\title{
Mammalian conserved ADAR targets comprise only a small fragment of the human editosome
}

\author{
Yishay Pinto, Haim Y Cohen and Erez Y Levanon*
}

\begin{abstract}
Background: ADAR proteins are among the most extensively studied RNA binding proteins. They bind to their target and deaminate specific adenosines to inosines. ADAR activity is essential, and the editing of a subset of their targets is critical for viability. Recently, a huge number of novel ADAR targets were detected by analyzing next generation sequencing data. Most of these novel editing sites are located in lineage-specific genomic repeats, probably a result of overactivity of editing enzymes, thus masking the functional sites. In this study we aim to identify the set of mammalian conserved ADAR targets.

Results: We used RNA sequencing data from human, mouse, rat, cow, opossum, and platypus to define the conserved mammalian set of ADAR targets. We found that the conserved mammalian editing sites are surprisingly small in number and have unique characteristics that distinguish them from non-conserved ones. The sites that constitute the set have a distinct genomic distribution, tend to be located in genes encoding neurotransmitter receptors or other synapse related proteins, and have higher editing and expression levels. We also found a high consistency of editing levels of this set within mice strains and between human and mouse. Tight regulation of editing in these sites across strains and species implies their functional importance.

Conclusions: Despite the discovery of numerous editing targets, only a small number of them are conserved within mammalian evolution. These sites are extremely highly conserved and exhibit unique features, such as tight regulation, and probably play a pivotal role in mammalian biology.
\end{abstract}

\section{Background}

The canonical post-transcriptional modification of adenosine to inosine (A-to-I) deamination is catalyzed by enzymes of the ADAR family, which bind to double-stranded RNA (dsRNA) [1-3]. This reaction takes place in a wide variety of organisms of the metazoan lineage [4-9]. A-to-I substitution causes the intracellular translation machinery to identify inosine (I) as guanosine (G), and thus, can lead to protein diversification. In addition to creating synonymous and non-synonymous codon changes, several additional functional and regulatory implications were also found for editing. RNA editing is involved in several processes, including: alteration of pre-mRNA splicing by the creation or elimination of splice sites [10-12]; RNA degradation [13,14]; viral RNA replication [15]; nuclear retention of transcripts [16]; miRNA regulation [17,18]; and protein susceptibility to proteolytic cleavage [19].

\footnotetext{
* Correspondence: erez.levanon@biu.ac.il

Mina and Everard Goodman Faculty of Life Sciences, Bar-llan University, Ramat Gan, Israel
}

Vast amounts of sequencing data have become available over the last few years. Consecutive computational approaches were developed to identify novel RNA editing sites, taking advantage of the available large RNA sequencing (RNA-seq) datasets. Such analysis is generally performed by comparing DNA to RNA sequences [12,20-24]. Editing events are detected as A-to-G mismatches between the reference genome and the RNA reads. Although the concept is simple, these approaches are very susceptible to false positives due to a low signal-to-noise ratio, caused by sequencing and alignment errors and mismatches derived from somatic mutations and polymorphisms in the population [22,25-27]. The current rapid progress in sequencing technologies, led to the publication of a huge number of editing sites, more than a million in human [20,28], and thousands of additional ones in mouse $[4,29]$ and Drosophila [5,30]. Most of these sites are consequences of double-stranded RNA structures formed by inverted, usually lineage specific, repeats (for example, Alu pairs $[12,31-33]$ in human, and B1 in mouse [29]). Yet, it is 
not clear which of the sites have functional importance, and how many are only the outcomes of residual ADAR activity, with no selective advantage.

Although most of the sites are located in genomic repeats, and seem to have no functional importance, RNA editing is an essential process, as $\mathrm{ADAR} 1^{-/-}$and $\mathrm{ADAR}^{-/-}$mice exhibit embryonic and postnatal lethal phenotypes, respectively [34,35], and editing is involved in several key cellular functions and diseases [35-39]. Indeed, important biological functions were assigned to several recoding sites (editing sites that change the translated protein sequence). Most of these established sites are located in neuronal genes, such as the editing site in the glutamate receptor (GLUR2) gene that leads to a non-synonymous substitution (Q607R), which takes place in glutamatergic neurons in close to $100 \%$ of the transcripts (100\% editing levels) [40]. Other examples are the cluster of five editing events in the serotonin receptor $5 \mathrm{HT}_{2 \mathrm{C}} \mathrm{R}$ which regulates mood, appetite, and behavior [41-43], and the editing event in the voltage-gated potassium channel, Kcna1 [44].

In this study, we wished to identify from within the large number of novel editing sites, those sites that became important over the course of mammalian evolution. In order to achieve this goal, we used evolution as the key selection tool, looking for sites that are common in several lineages, which suggest that they were functionally selected. Creating such a catalog of selected editing sites will have a remarkable effect on functional editing research, since it illuminates the few 'meaningful' sites, by not only defining the functional sites, but also by suggesting a method to define them.

Surprisingly, we found that the number of such conserved editing sites is extremely small, and probably only a limited set of such functionally important editing sites exist. We found that the plethora of sequencing data did not contribute much to the discovery of novel conserved sites, as most of the functionally important sites were known before the next generation sequencing revolution. Nevertheless, we were able to discover, based on their extreme conservation, the few editing sites that probably play a pivotal role in mammalian biology. In addition, our results demonstrate that editing, in parallel to the established mutational processes that shape genomes, add another layer of variability and complexity that can be spatiotemporally regulated.

\section{Results}

The conserved editing set is small

Most of the known editing sites seem to be located in lineage-specific regions, mainly in inverted repeats as is the case in the human [20,28], mouse [4], and fruit fly [5]. Only very few sites are known to be conserved across large evolutionary distances. For example, only one site was found to be conserved between human and Drosophila fly $[45,46]$ (probably due to convergent evolution) and only a handful of sites were found to be conserved between human and mouse, so far [21]. In the last few years, the total number of known human editing sites jumped by several orders of magnitude; thus, many expected that the number of functional sites would grow at the same rate.

In order to build a comprehensive and updated dataset of conserved mammalian editing sites, we collected all available RNA editing sites from recent RNA-seq studies of both human and mouse. This dataset contains a total of 1,432,743 human sites [20,28,47] and 10,210 [4,47-50] mouse sites. All were found by aligning large sets of RNA sequences, in an unbiased manner, to the matched genomes. In order to find sites that are highly conserved between species, we retrieved for each site the $80 \mathrm{bp}$ flanking genomic sequence (40 nucleotides upstream and 40 downstream) and aligned each of the human sequences to all mouse sequences using the standard BLAST [51] alignment tool. We filtered out sites below stringent alignment thresholds (an identity of at least 70 of the 81 nucleotides), and retained only sites in which the A-to-G mismatch appears in both human and mouse at the same position (see Methods). Applying this straightforward procedure resulted in 59 evolutionary selected sites (ESS) (Table 1, Figure 1A-B and in Additional file 1: Table S1). Surprisingly, we found that the number of sites in the ESS is very small $(0.004 \%$ of human sites) and increased only slightly in recent years, while the total number of sites grow by several orders of magnitude (Figure 1C). We found that this set was hardly affected by changing the alignment parameters. In addition, we obtained similar results when we used the UCSC lift over tool, which converts coordinates between genomes [52] (see Additional file 1: Table S2), suggesting that this set is coherent and unique (only one additional coding target in the GLI gene was added by this method). The sensitivity of this approach appears to be very high as the set contains all the previously well characterized sites. Even though there was a dramatic increase in the number of identified editing sites over the last few years, the number of conserved sites remains low. In order to estimate the selectivity of our approach, we calculated the odds of two unrelated genomic events taking place by chance at the same genomic position, in both the human and mouse genomes. For this purpose, we applied the same above procedures on human and mouse SNPs located on chromosome X. This resulted in only 1.8 conserved SNPs (normalized to a database size of 443,366 SNPs in human and 453,726 in mouse) retrieved by the same BLAST parameters. Thus, we measured the signal-to-noise ratio at the editing set to be at least 32 (Figure 1D). Taken together, these results indicate that our set of sites is both robust and accurate. 
Table 1 Mammalian evolutionarily conserved sites

\begin{tabular}{|c|c|c|c|c|c|c|}
\hline & chr & Position & Strand & Gene & Region & ref_id:nucleotide cange:aa change \\
\hline 1 & chr1 & 160302244 & - & COPA & CDS & NM_001098398:c.A490G:p.1164V \\
\hline 2 & chr11 & 105804694 & + & GRIA4 & CDS & NM_000829:c.A2293G:p.R765G \\
\hline 3 & chr11 & 105815132 & + & GRIA4 & intron & \\
\hline 4 & chr11 & 105816106 & + & GRIA4 & intron & \\
\hline 5 & chr11 & 105816129 & + & GRIA4 & intron & \\
\hline 6 & chr11 & 105816145 & + & GRIA4 & intron & \\
\hline 7 & chr11 & 105816160 & + & GRIA4 & intron & \\
\hline 8 & chr12 & 5021742 & + & KCNA1 & CDS & NM_000217:c.A1198G:p.|400V \\
\hline 9 & chr13 & 46090371 & + & COG3 & CDS & NM_031431:c.A1903G:p.1635V \\
\hline 10 & chr14 & 26917530 & - & NOVA1 & CDS & NM_006489:C.A1087G:p.S363G \\
\hline 11 & chr14 & 101506074 & + & mir376C & microRNA & \\
\hline 12 & chr17 & 43045220 & - & C1QL1 & CDS & NM_006688:c.A197G:p.Q66R \\
\hline 13 & chr19 & 47152854 & - & DACT3 & CDS & NM_145056:c.A775G:p.R259G \\
\hline 14 & chr2 & 20450819 & - & PUM2 & 3'UTR & \\
\hline 15 & chr2 & 21233202 & - & APOB & CDS & NM_000384:c.C6538G:p.Q2180stop \\
\hline 16 & chr2 & 210835613 & + & UNC80 & CDS & NM_032504:c.A7990G:p.S2664G \\
\hline 17 & chr20 & 36147533 & - & BLCAP & CDS & NM_001167821:c.A44G:p.K15R \\
\hline 18 & chr20 & 36147563 & - & BLCAP & CDS & NM_001167821:c.A14G:p.Q5R \\
\hline 19 & chr20 & 36147572 & - & BLCAP & CDS & NM_001167821:c.A5G:p.Y2C \\
\hline 20 & chr20 & 36148080 & - & BLCAP & intron & \\
\hline 21 & chr20 & 52104918 & + & TSHZ2 & 3'UTR & \\
\hline 22 & chr21 & 30953750 & - & GRIK1 & CDS & NM_175611:c.A1862G:p.Q621R \\
\hline 23 & chr21 & 34922801 & + & SON & CDS & NM_032195:c.A1264G:p.T422A \\
\hline 24 & chr21 & 34923319 & + & SON & CDS & NM_032195:c.A1782G:p.L594L \\
\hline 25 & chr21 & 46595620 & + & ADARB1 & intron & \\
\hline 26 & chr3 & 53820892 & + & CACNA1D & CDS & NM_001128839:c.A4791G:p.11597M \\
\hline 27 & chr3 & 58141801 & + & FLNB & CDS & NM_001164319:c.A6815G:p.Q2272R \\
\hline 28 & chr3 & 62423807 & - & CADPS & CDS & NM_183393:c.A3512G:p.E1171G \\
\hline 29 & chr4 & 57976234 & - & IGFBP7 & CDS & NM_001253835:c.A284G:p.K95R \\
\hline 30 & chr4 & 57976286 & - & IGFBP7 & CDS & NM_001253835:c.A232G:p.R78G \\
\hline 31 & chr4 & 158257875 & + & GRIA2 & CDS & NM_000826:c.A1820G:p.Q607R \\
\hline 32 & chr4 & 158257879 & + & GRIA2 & CDS & \\
\hline 33 & chr4 & 158258136 & + & GRIA2 & intron & \\
\hline 34 & chr4 & 158258137 & + & GRIA2 & intron & \\
\hline 35 & chr4 & 158281294 & + & GRIA2 & CDS & NM_000826:c.A2290G:p.R764G \\
\hline 36 & chr5 & 156736808 & + & CYFIP2 & CDS & NM_001037332:c.A958G:p.K320E \\
\hline 37 & chr6 & 34100903 & - & GRM4 & CDS & NM_000841:c.A371G:p.Q124R \\
\hline 38 & chr6 & 44120349 & + & TMEM63B & CDS & NM_018426:c.A1856G:p.Q619R \\
\hline 39 & chr6 & 102337689 & + & GRIK2 & CDS & NM_001166247:c.A1699G:p.1567V \\
\hline 40 & chr6 & 102337702 & + & GRIK2 & CDS & NM_001166247:c.A1712G:p.Y571C \\
\hline 41 & chr6 & 102372589 & + & GRIK2 & CDS & NM_001166247:c.A1862G:p.Q621R \\
\hline 42 & chr6 & 102372630 & + & GRIK2 & intron & \\
\hline 43 & chr6 & 102374616 & + & GRIK2 & intron & \\
\hline 44 & chr6 & 102374643 & + & GRIK2 & intron & \\
\hline
\end{tabular}


Table 1 Mammalian evolutionarily conserved sites (Continued)

\begin{tabular}{|c|c|c|c|c|c|c|}
\hline 45 & chr6 & 150093334 & + & PCMT1 & intron & \\
\hline 46 & chr8 & 103841636 & - & AZIN1 & CDS & NM_148174:c.A1099G:p.S367G \\
\hline 47 & chr8 & 103841637 & - & AZIN1 & CDS & NM_148174:c.A1098G:p.E366E \\
\hline 48 & chr9 & 97847739 & + & mir23B & microRNA & \\
\hline 49 & $\operatorname{chr} x$ & 114082682 & + & HTR2C & CDS & NM_000868:c.A466G:p.1156V \\
\hline 50 & $\operatorname{chr} x$ & 114082684 & + & HTR2C & CDS & NM_000868:c.A468G:p.1156M \\
\hline 51 & $\operatorname{chr} x$ & 114082689 & + & HTR2C & CDS & NM_000868:c.A473G:p.N158S \\
\hline 52 & $\operatorname{chr} x$ & 114082694 & + & HTR2C & CDS & NM_000868:c.A478G:p.1160V \\
\hline 53 & $\operatorname{chr} x$ & 122598962 & + & GRIA3 & CDS & NM_000828:C.A2323G:p.R775G \\
\hline 54 & $\operatorname{chr} x$ & 122598998 & + & GRIA3 & intron & \\
\hline 55 & $\operatorname{chr} x$ & 135111055 & + & SLC9A6 & intron & \\
\hline 56 & $\operatorname{chr} x$ & 135111070 & + & SLC9A6 & intron & \\
\hline 57 & $\operatorname{chr} x$ & 151358319 & - & GABRA3 & CDS & NM_000808:c.A1026G:p.1342M \\
\hline 58 & $\operatorname{chr} x$ & 153579737 & - & FLNA & intron & \\
\hline 59 & $\operatorname{chr} x$ & 153579950 & - & FLNA & CDS & NM_001456:C.A6998G:p.Q2333R \\
\hline
\end{tabular}

List of conserved editing sites. Coordinates are based on the genome version GRCh37/hg19. For each site, the table includes the following information: chromosome, genomic coordinate, strand, gene name, genomic compartment, RefSeq id (if available), editing transformation, and coordinate related to the Refseq ID, and amino acid change (for the same Refseq ID).

\section{More data do not guarantee a greater number of ESS}

As sequencing data accumulated, the total number of identified editing sites increased as well. However, we found that the number of the evolutionarily selected editing sites did not increase when new sequencing data were added. Even though the set is rather small, its sensitivity (recall) and specificity rates seem to be strikingly high. The high recall rate was confirmed as the set contains all the conserved functional sites known so far. To measure the specificity of the ESS, we estimated the effect of accumulating a species-specific RNA editing dataset on the ESS size. In order to demonstrate that the size of the ESS does not dramatically change as the data accumulate, we found that even a small fraction of the available data is sufficient to retrieve most of the ESS. We used data from 15 mouse strains [4] to build a site accumulation curve (Figure 2A, Additional file 2: Figure S1). We found that for any random choice of two strains, we retrieved at least $94 \%$ of the sites (and only $72 \%$ of all other sites), and reached full saturation after including only six strains. Thus, the ESS is less sensitive to dataset enlargement compared to the set of all other mouse sites.

There are two reasons for explaining the few cases in which the editing signal was not detected in a specific mouse strain. The first is the low read depth (low expression), which makes measurement of editing levels difficult. The second explanation is editing levels under the detection threshold (or no editing at all). There is clear distinction between the conserved and the non-conserved sites: while there were only a few cases of sufficient coverage with no signal for editing in the conserved sites, there were a higher proportion of sites with sufficient coverage but no editing signal for the non-conserved ones. Many of the latter are not reproduced across different samples [53]. Visualization of the editing signal per strain by heat-maps is shown in Figure 2B-D. Our findings suggest that the selected sites tend to be edited in almost all mouse strains, while the non-conserved sites showed a weaker tendency for such consistency.

\section{Editing profiles for ESS in an additional four mammals}

We analyzed RNA-seq data to find editing levels for the matched position of the ESS in four additional mammals: rat, cow, the non-placental marsupial opossum, and the monotrem platypus $[54,55]$. They were chosen based on the quality of their genomes, their evolutionary distance, and the availability of the necessary RNA-seq data. Although we analyzed only limited RNA-seq data per organism, we observed strong evidence for editing in the matched ESS for these species (Additional file 1: Table S3). In the rat transcriptome, $93.7 \%$ (45 out of 48 ) of the sites with a minimal coverage's ( $>5$ reads) exhibit evidence for editing. Similarly, $100 \%$ (23 out of 23) of sites with such coverage in the cow are edited. As expected, the very evolutionary distant opossum and platypus exhibit somewhat less evidence of editing with approximately $67 \%$ (21 out of 31 and 16 out of 24, respectively) of covered sites. These results are very strong, since we used only one or two RNA-seq datasets per organism. For example, in a single RNA-seq, derived from human brain, only $83 \%$ of the sites were found to be edited (Additional file 1: Table S4). We failed to find evidence for matched editing at only four 


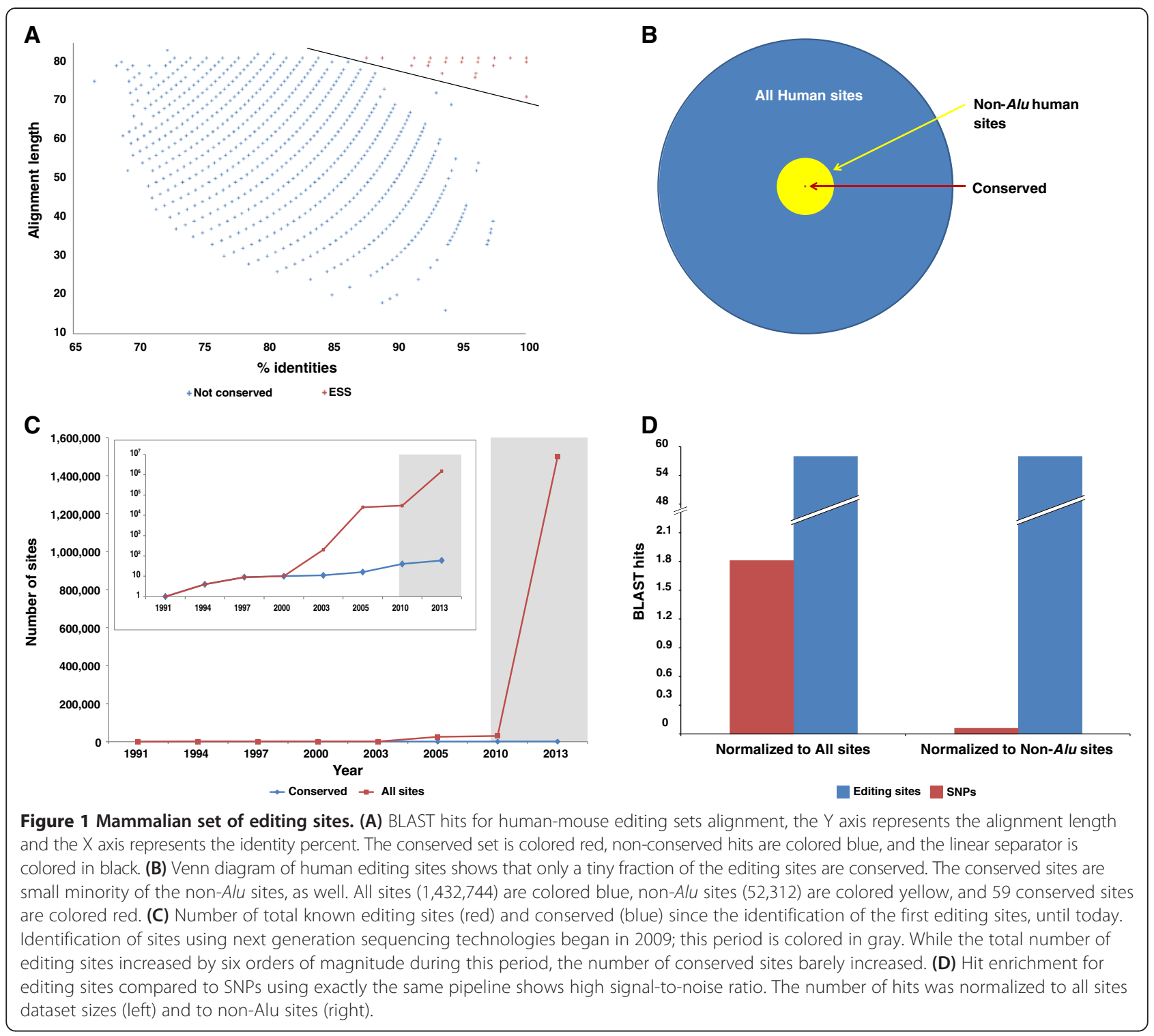

sites (2 intronic in Gria4, and 2 coding Dact3 and C1ql1) in any the tested organisms, mainly due to low coverage for those sites.

\section{Genomic location of ESS}

Our results indicate that although both human and mouse transcriptomes contain a large number of editing sites, only a small group of the sites are shared as far back as the common ancestor of rodents and primates. These sites have different genomic characteristics versus the non-conserved sites. As expected, most of the sites in the ESS are located in coding regions (37 sites, 64\%), (Figure 3A) and an absolute majority of these sites (35 sites, 94\%) lead to non-synonymous mutations, which is a driving force in adaptive evolution (Figure 3B). The only two synonymous sites are located proximately to other non-synonymous site in the SON and AZIN genes, and their editing may be only a residual activity of ADAR near the main site.

We also found a relatively high number of sites located in introns (17 sites, 29.3\%). In contrast to exons that have a clear potential for evolutionary benefit, such as amino acid changes, introns are considered as fitness-neutral in nature, and the reason they are evolutionarily conserved might look enigmatic. One probable explanation for the editing sites found in introns, is that the intronic sites are located in exon complementary sequence (ECS), a genomic region needed for dsRNA structure formation, which is required for ADAR binding [56]. Indeed, we found that 13 of the 17 intronic sites $(76.4 \%)$ are located in genes that have recoding events as well (while only $11.1 \%$ for the control non-conserved intronic sites). Furthermore, by 


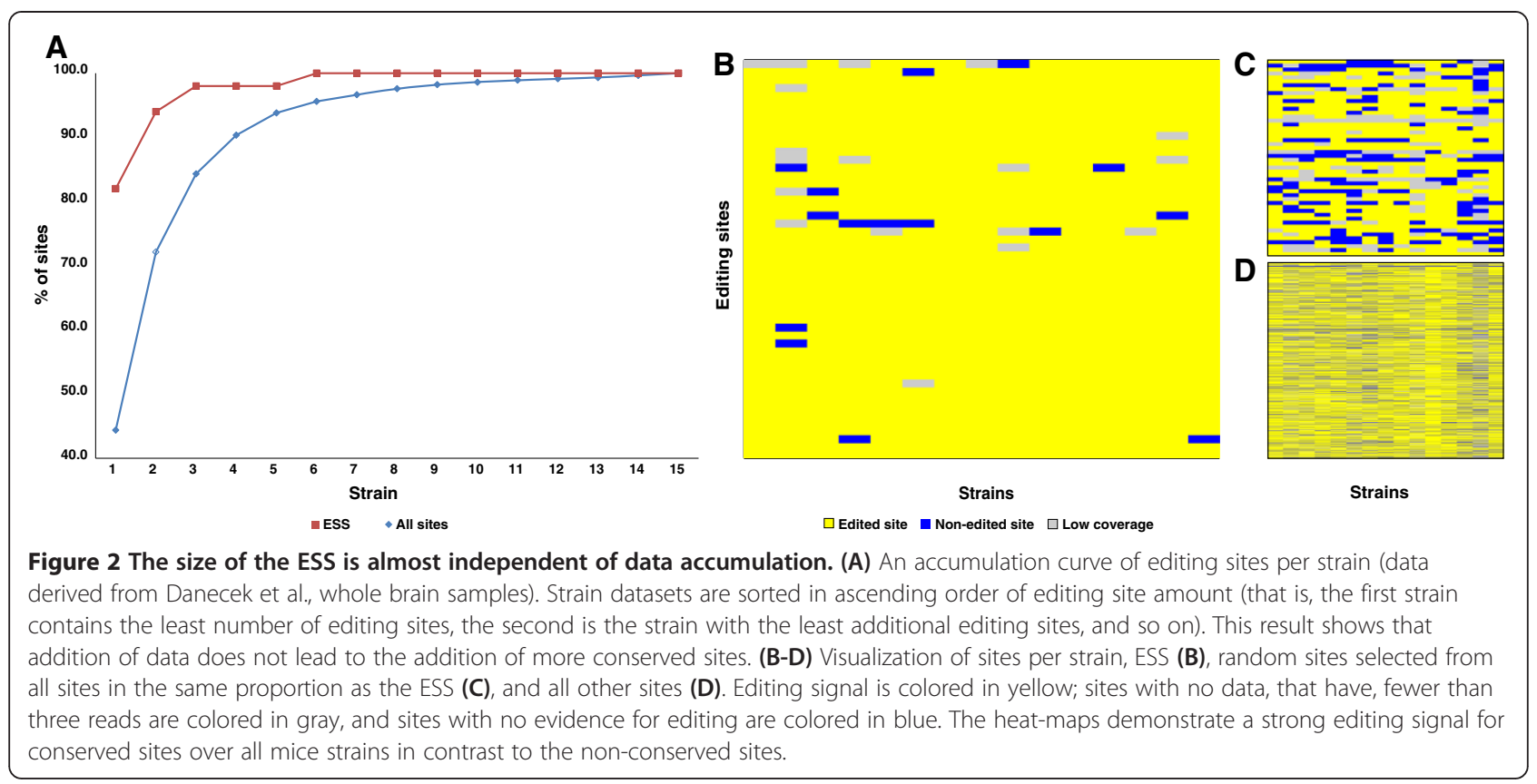

using secondary RNA structure prediction software (mfold) [57] for those sites (Figure 3C-E), we were able to confirm that 11 of 13 sites are located in complementary regions of other conserved editing sites (Additional file 2: Figure S2). In one interesting case, we found that the dsRNA structure was formed between two inverted introns. This structure contains a cluster of five proximate sites located in the GRIA4 gene. The extreme conservation of the two inverted introns and the five sites they harbor suggests that at least one of them has a functional role (Additional file 2: Figure S2A). Identification of the intronic ECS of a site is intrinsically important to study the function of the editing event in the coding region. As demonstrated in the past for several editing sites $[10,44,58,59]$, a transgenic mouse with the unedited mRNA transcript can be generated by removal of the intronic ECS sequence. The identification of new ECS will allow functional studies of the corresponding editing sites.

Three additional sites were found in non-coding regions. Two sites are located in miRNAs (although short noncoding RNA sequences are usually depleted in RNA-seq libraries). The first one, previously published [18], is located within the seed region of mir-376c (Additional file 2: Figure S3). This editing event can alter the predicted targets of this miRNA. The second, an un-annotated conserved site, is located in mir-27b (Additional file 2: Figure S3). This miRNA is known to regulate many key processes such as lipid metabolism, inflammation and angiogenesis $[60,61]$. The third site is located within the 3' UTR of the TSHZ2 gene. The site is located within the highly conserved $200 \mathrm{nt}$ region, and we found a cluster of another four sites $150 \mathrm{nt}$ upstream to this site in the mouse dataset. Notably, 11 of the sites are annotated as SNPs in dbSNP. Such erroneous annotation has been demonstrated in many of the previously identified editing sites [62,63], as sequences undergoing A-to-I RNA editing could be incorrectly identified as an A/G SNP. Former methods to discover SNPs used RNA sequences as well, and thus may be subject to this error. Indeed, the annotation of such SNP at dbSNP indicates that this SNP was detected by analysis performed on a cDNA library.

\section{Motif sequence}

Previous studies indicated that ADARs have a sequence preference for $G$ depletion on the upstream nucleotide to the editing site and have excess of $G$ at its 3 ' base. We compared the nucleotide frequency for both the ESS and a control set (all human non-Alu sites). Although both sets adhered to the previously published neighbor preferences [64,65] (Figure 4), the motif signal of the ESS was stronger than the control, probably due to a purifying selection effect. Similar results were found in the mouse set, as well. This result supports the idea that the large un-conserved human and mouse sets do contain mostly genuine editing sites, but only very few are evolutionarily selected.

Conserved sites have higher editing and expression levels We calculated the distribution of editing levels for the ESS and for all of the other previously published [4] sites in mouse (Figure 5A). Editing levels of a site are defined by the following ratio: (the number of $\mathrm{G}$ reads)/(the total number of $A$ and $G$ reads) of the base calling at the specific editing position. We found an over-representation of sites 

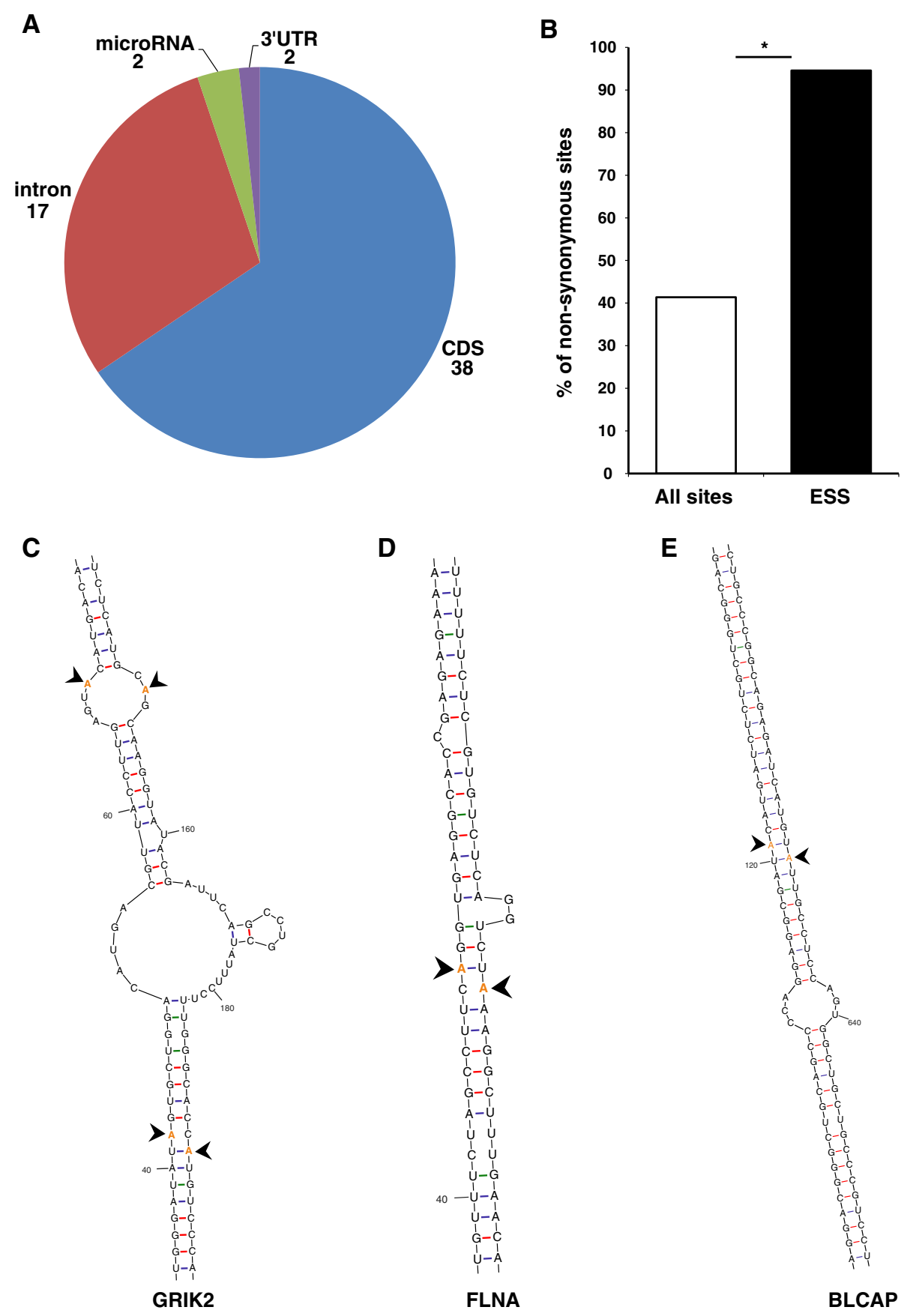

Figure 3 Most of the ESS sites are located in a coding region or adjacent to such a site. (A) Genomic location of evolutionarily conserved sites. (B) Frequency of non-synonymous editing alterations in exonic sites for both groups demonstrates enrichment of sites that cause amino acid change in the ESS compared to the control (all other sites, $P<2 \times 10^{-11}$ calculated by Fisher's exact test). (C-E) Secondary structure shows spatial proximity of coding and intron sites of GRIK2 (C), FLNA (D) and BLCAP (E) genes; editing sites are highlighted in orange and marked by an arrow.

with high editing levels in the ESS compared to all other sites. Comparing the average editing levels revealed two-fold higher editing levels in the ESS (Figure 5B). High editing levels imply that the edited transcript is functional. In addition, we examined expression levels, by counting the coverage of each site. Here, again, we found that ESS sites have significantly higher expression levels than the remaining sites (Figure 5C-D), which also implies their significant function.

Further support for the idea that the ESS are subjected to strong selective pressure and are highly regulated is the consistency [66] of the editing levels between the 

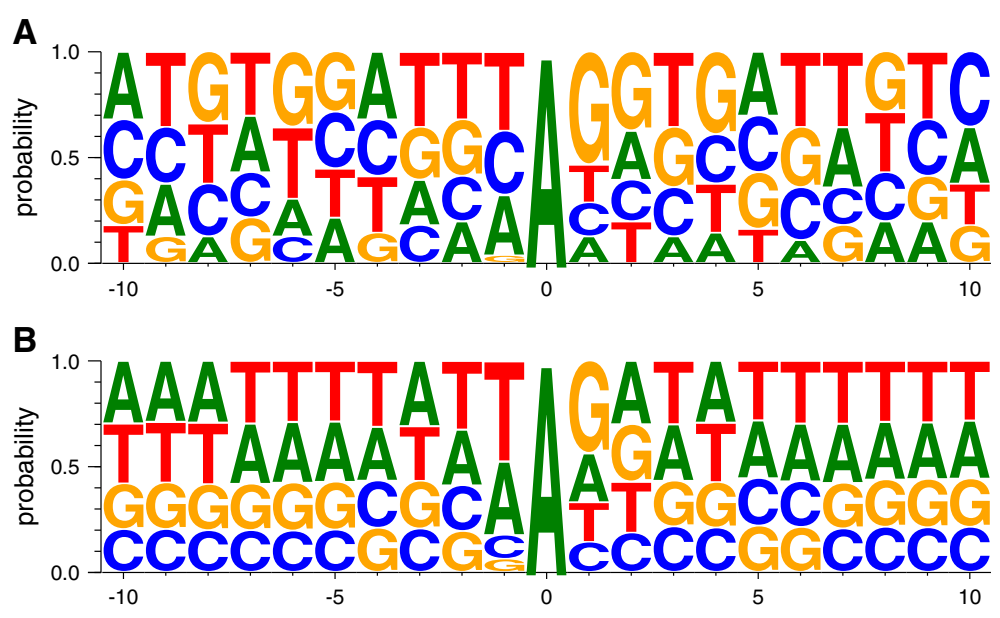

Figure 4 Neighbor preferences for ESS and all sites. Nucleotide frequency for ESS (A), and all non-Alu sites (B). Both signatures are in agreement with the ADAR motif.

different mouse strains. While the standard deviation between editing levels across 15 mice strains was rather high at the non-conserved sites, the same calculation revealed a striking conservation of editing levels in the ESS (11.1 for ESS, 18.4 for other sites, $P=4.6 \times 10^{-8}$; two-tailed Student's t-test, Figure $5 \mathrm{E})$. Furthermore, we found a very high degree of similarity (correlation $r=0.93$ ) of editing levels between two separate, recently published editing datasets $[4,50]$ from mouse brain. In addition, we found consistent editing levels between human and mouse although millions of years of evolution separate them $(r=0.55$ for all the ESS, and $r=0.77$ for coding sites only, Additional file 2: Figure S4 and Additional file 1: Table S1). These findings indicate the ESS were selected due to the function they provide to the edited transcript. In addition, we made an editing level map of all the conserved sites in 16 different human tissues, by analyzing the available humanbody-map RNA-seqs (Additional file 1: Table S4). As expected, we found that the most highly edited tissue is the brain. This result is consistent with the function of the conserved edited genes and with the fact that the majority of the mouse RNA-seq data originated from the brain. Although in general the editing levels of most of the sites are low [28] and therefore have a limited effect on the final protein product, the editing levels of the conserved sets are rather high and are similar for both human and mouse (average of $51.5 \%$ and $51.4 \%$, respectively). For 27 sites in human and 25 in mouse (19 in both) the edited version is dominant and has more transcripts than the genomic encoded ones $(>50 \%$ editing levels). A list of editing levels in human and mouse is provided in Additional file 1: Table S1.

The most commonly edited genes are BLCAP and IGFBP7, which are edited in all 16 tissues, while COG3,
TSHZ2, SON, COPA, PUM2, AZIN1, and FLNA genes are found to be edited in at least 10 tissues. All the sites are located in coding sequences or in the 3' UTR. This finding supports the hypothesis that coding sites are the main functional targets of ADARs, while intronic editing events probably represent residual ADAR activity. By counting the total number of edited reads for each site, assuming that the number of reads is correlated to expression levels, we found that the K/R site in IGFBP7 is the primary 'consumer' of ADARs in the human brain.

\section{Functional impact of editing}

RNA editing increases the diversity of the genomic outcome in a specific locus by creating A or $G$ variants. Specific gene families such as ion channels can utilize this capacity for rapid diversity by recoding genomic information. Indeed, we found that our set is enriched with $\mathrm{GO}$ terms that are related to neuron-specific functions, such as synaptic transmission, ion transport, and genes involved in neuroactive ligand-receptor interaction pathway (Additional file 1: Table S5). The editing of genes that encode proteins involved in neuronal excitability such as ion channels and transporters creates plasticity that can be used in response to environmental changes [67]. Comparing the ESS genes and proteins with other human genes and proteins revealed an unexpected result. The edited transcripts tend to be significantly longer than the average length of unedited human transcripts (5,674 bp in the ESS, 2,750 bp for human average transcripts, similar results for mouse). Similarly, the same trend was observed in the protein length (1,098 aa in the ESS and 328 for all human proteins). We have no apparent explanation for this phenomenon other than that longer genes have greater chances of being co-transcriptionally edited. 


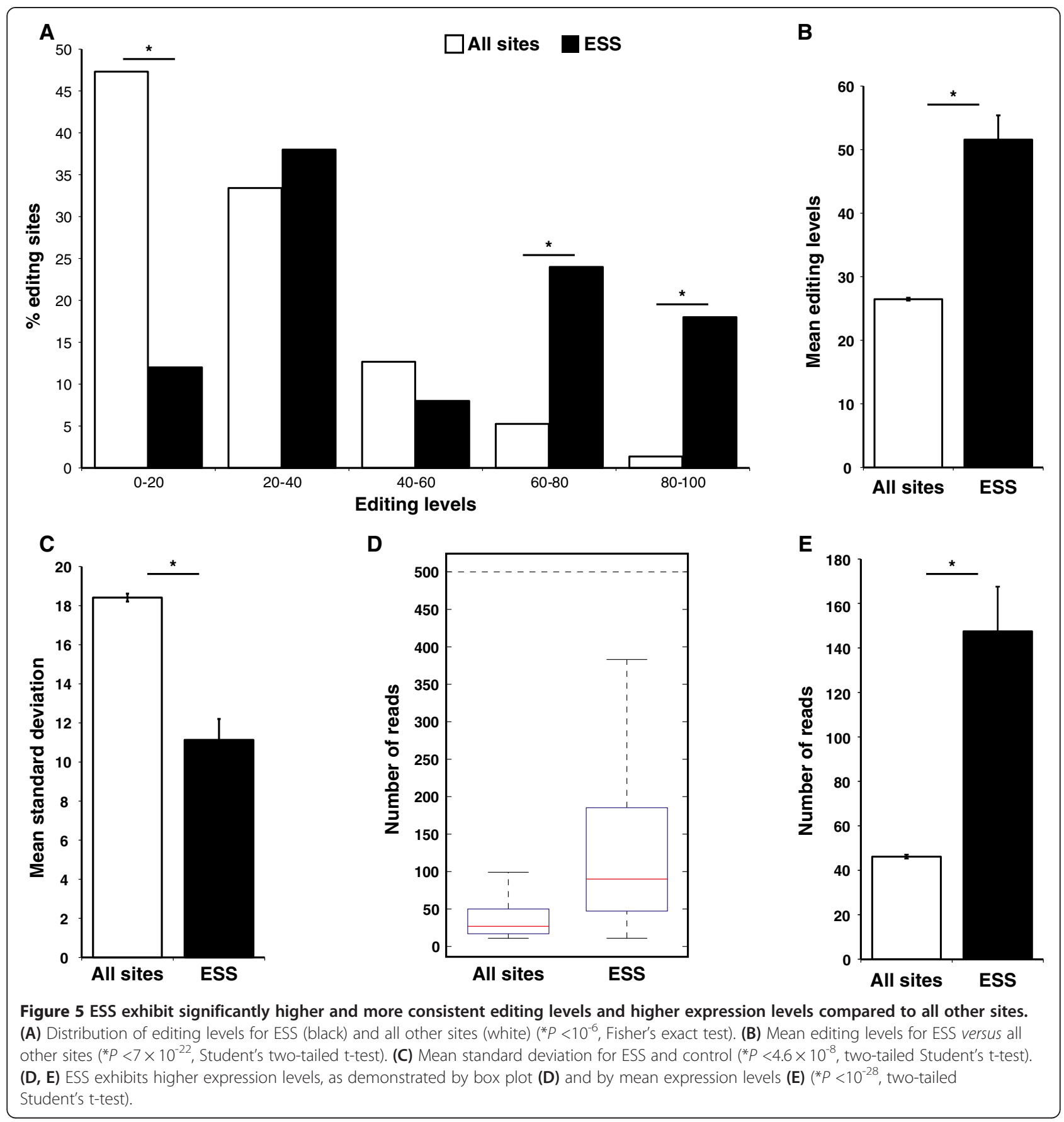

Interestingly, the main $\mathrm{C}$-to- $\mathrm{U}$ editing target of APOBEC1, the apoB transcript, encodes for an extremely long protein (4,563AA). This site is located in one of the largest encoding exons of the human genome $(>7,500 \mathrm{bp})$, further strengthening the connection between editing and long transcripts.

Editing tends to preserve an ancient version of the genome An additional benefit of RNA editing is the ability to mitigate evolutionary changes, thereby maintaining evolutionary equilibrium. All editing positions have, by definition, a genomic adenosine. This adenosine is not always found in the matched position in genomes of other organisms. In the instances where there was no adenosine at the matched position, we found a majority of cases with ' $G$ ' hardwired at the counterpart genomic positions. Interestingly, when comparing the prevalence of genomic G in human and mouse sites, we found an asymmetric phenomenon. While $26 \%$ of all human editing sites with matching position (total of 12,937 sites) harbor a $G$ in the 
mouse genome, only $18 \%$ (out of 1,083 such sites) of mouse editing sites harbor $\mathrm{G}$ in the human genome (Figure 6, $P=2.1 \times 10^{-7}$, Fisher's exact test). As a control, we applied the same procedure to adjacent but non-edited As. Here, no significant trend was found, and a similar percent (19\%) of the human and mouse sites have a G at the corresponding position, suggesting this result is specific for edited adenosine. These results suggest that in the majority of cases, editing serves as a mechanism to compensate for a loss of phenotype caused by G-to-A evolution. This versatile reversion may occur in only part of the transcripts in parallel to the non-edited version and in a tissue-specific manner. Thus, editing allows the functional co-existence of two independently evolved genome versions. Furthermore, our results suggest that in addition to

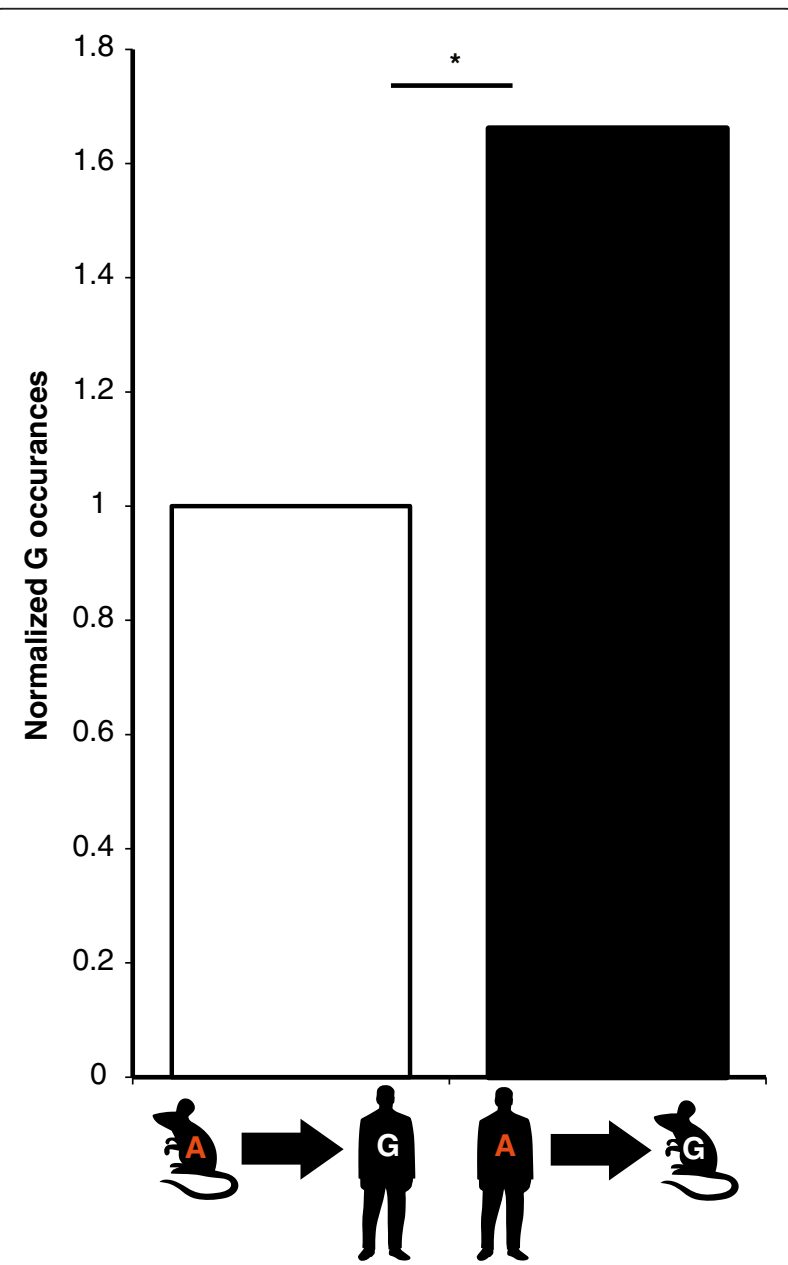

Figure 6 A-to-I editing as a mechanism for the reversion of G-to-A evolution. All mouse editing sites were converted to human genome coordinates. G-to-A ratio was calculated and fixed as 1 (left). All human editing sites were converted to mouse genome coordinates; G-to-A ratio was calculated and normalized (right), exhibiting 1.66-fold enrichment compared to the mouse-to-human conversion. ( ${ }^{*} P=10^{-7}$, Fisher's exact test). the ESS, there are additional functional editing sites in humans that have a $\mathrm{G}$ in the mouse genome (Additional file 1: Table S6), and therefore, were not included in this screen.

\section{Editing is involved in exonization of the LINE retroelement}

Although most of the conserved editing sites are located in conserved regions of a protein (or in the ECS region), we found at least one editing site which seems to be involved in exonization of a retroelement (Figure 7A). In this unique case (SLC9A6), we found two editing sites located in a newly emerged exon derived from an L2 repeat. This is one of the first documented cases of preserved LINE exonization throughout mammalian evolution [68]. Since the complementary LINE that enables the editing is also conserved, a rare event by itself, we can assume that all three rare events (exonization of LINE, conservation of two LINEs, and two conserved editing events) are related, suggesting that editing was a driving force for the exonization and conservation of this element. The close proximity of editing to the splicing site provides additional supporting evidence for the involvement of editing in this exonization. The alternatively spliced exon is located in the SLC9A6 gene which has been implicated in several disorders causing mental retardation [69]. The gene product is the NHE6 protein, a hydrogen sodium exchanger. This channel controls the $\mathrm{pH}$ inside endosomes, which is important for the proper function of these compartments. Moreover, this ion exchanger was found to regulate clathrin dependent endocytosis of transferrin. The insertion of the alternative exon enlarges the protein by 30 amino acids, starting at position 507. The exon inclusion creates a longer C-terminal cytoplasmic tail. The editing sites convert the arginine (basic polar, positive side chain) at positions 511 and 514 to the non-polar and neutral glycine (R511G and R514G). Validation of the presence of this exon and editing sites is shown in Figure 7B. We believe this case is a unique example in which editing contributes to creation of new functional units.

\section{Insights from the vertebrate set}

We also analyzed RNA-seq data to identify RNA editing candidates in zebrafish (see Methods). We used the same method to find conserved sites between human and mouse and zebrafish, which is one of the most evolutionarily distant vertebrate relatives of human for which genome and transcriptome data are available. We found 17 ESS sites that are also conserved in zebrafish (Additional file 1: Table S7), and most of them (10) are located in glutamate receptors. This enrichment is consistent with the high repertoire of glutamate receptors found in the zebrafish genome. The genomic sequence of the ESS sites is highly conserved across a diverse set of distant mammals (mouse, dog, and opossum) and includes most of the intronic ECS 


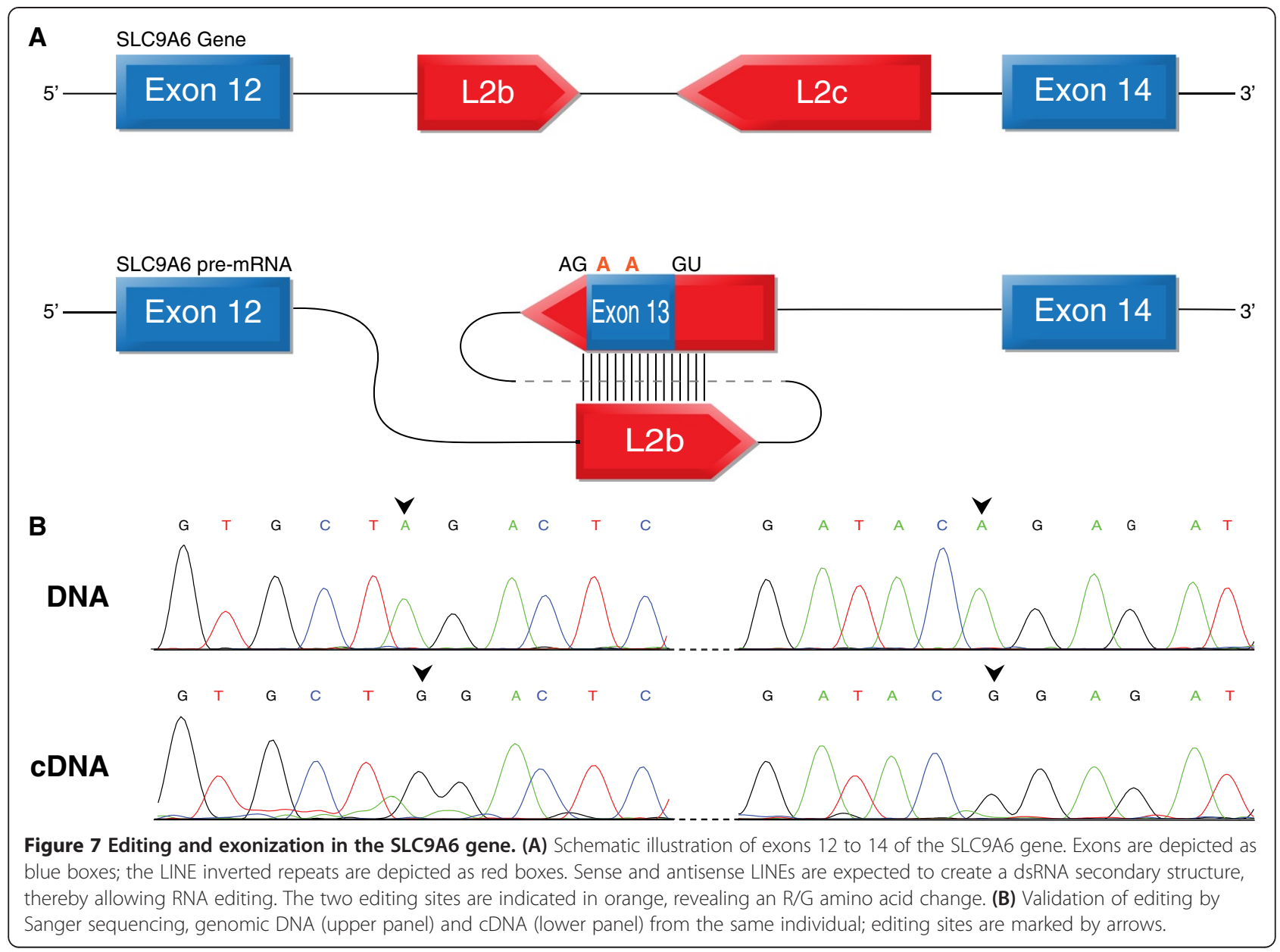

regions as well, suggesting that editing also takes place in these mammals too. Moreover, most of the sequences (45 sites, 76\%) are also highly conserved in non-mammalian vertebrates (chicken and zebrafish, see Additional file 1: Table S8).

\section{Non-conserved editing sites}

The large editing datasets we used for human and mouse were compiled from transcriptome wide analysis, which is subject to a high false positive rate, as previously described [20]. But, while the false positive rate in Alu regions is relatively low, the contamination of artifacts in nonrepetitive regions is very high; in some cases the noise levels in these regions are even higher than the A-to-G signal [20]. Knowing that the non-conserved set is noisy, allows us to describe it only in general terms. Unlike the conserved one, this set exhibits inconsistent editing events across samples (Figure 2). In addition, the sites in this set are usually located in fitness neutral regions, as $>90 \%$ of sites are located in introns and a majority of the coding sites lead to synonymous substitution (Figure 3B). Moreover, sites in this set exhibit low and variable editing levels (Figure 5B-C), and relatively low expression levels, as well (Figure 5D-E). Additional evidence for the stochastic nature of editing at these sites in this set, is that only $13.9 \%$ of the human specific sites exhibit an editing signal in any of the 16 tissues represented in the human body map, while a majority of the ESS (64.3\%) show clear signal for editing in this dataset (Additional file 1: Tables S4 and S9).

A list of non-synonymous non-conserved sites is provided in Additional file 1: Table S10.

\section{Discussion}

In this study, we defined, for the first time, the mammalian RNA editing set. Our results indicate that the conserved mammalian set of editing sites is extremely small and has distinct characteristics compared to the non-conserved sites. The conserved set has a unique genomic regional distribution, and has higher and more consistent editing levels, and higher expression levels than the non-conserved sites. Our results support the claim that only dozens of conserved functional editing sites exist, and negate the assumption that next-generation sequencing technologies 
will lead to the discovery of many additional novel conserved sites. One of those few targets, the SLC9A6 gene, demonstrates the evolution of an RNA editing site. This event is a result of dsRNA structure formation by the insertion of two inverted repeats, and the fixation of the locus across all mammalian lineages, probably due to the selective advantage provided by this sequence. A newly evolved site might be harmful, beneficial, or neutral. A harmful site will be eliminated quickly over the course of evolution. Conversely, a beneficial site will be conserved across evolution, and a neutral site will be eliminated at a slower evolutionary pace. It makes sense to assume that most of the lineage-specific sites are of neutral evolutionary fitness. Accordingly, it would be interesting to further investigate what advantage is provided by SLC9A6 editing.

Thirteen intronic sites were found to be located in a complementary sequence required for formation of dsRNA structure of another conserved editing site. When looking for the possible complementary regions for all sites, we were able to find the same strong evolutionary sequence conservation of more than 70 bp (out of 81) identity in 45 of the sites (See Additional file 1: Table S11). The remarkable conservation of two adjacent genomic regions for each site indicates that editing is the driving force for this conservation.

RNA editing can preserve a mutated genome version at the RNA molecule. This mechanism is beneficial, as it enables the simultaneous use of two evolved versions of an mRNA (the edited and the non-edited forms), at very low evolutionary cost [70]. Interestingly, we see clear evidence of editing to reverse evolutionary mutations, as opposed to editing being merely a 'stepping stone' for A-to-G genomic mutation.

Our studies revealed a comprehensive set of mammalian conserved sites. Yet, it is still possible that additional sites do exist and can be found if more samples from additional tissues (as most of the mouse data are derived from brain and liver) or genomes with higher coverage are used, or if less stringent criteria are used for conservation. However, our results suggest that adding more data or using relaxed parameters will not add many additional sites that are as highly edited and highly expressed. Therefore, we believe that this set is close to being inclusive, and if such additional sites do exist they are probably few in number.

\section{Conclusions}

We carried out the first systematic screening for conserved mammalian RNA editing. Although we explored comprehensive editing sets in human and mouse, we found that there are only a few RNA editing sites that are conserved between these species. Our results demonstrate that although additional RNA-seq data can lead to the identification of novel editing targets, they are unlikely to add many additional conserved sites. We found that the mammalian conserved set of editing sites is limited and has distinguishing characteristics that set these sites apart from others. The conserved sites have a unique genomic distribution and tend to be located in regions with evolutionary impact. Most of the conserved edited genes are related to neural-specific functions; yet, we found an editing signal in a wide variety of tissues. In addition, we found editing to be involved in the creation of a new functional unit by exonization of a repetitive element in the SLC9A6 gene. Finally, we showed that editing tends to preserve ancient genome versions and allows the co-existence of two evolved phenotypes.

\section{Methods}

\section{Creation of candidate RNA editing datasets}

The human RNA editing dataset was created by merging published datasets. These datasets were created by seeking RNA-DNA differences using RNA-seq analysis. The first dataset that we used contained all editing candidates identified by Ramaswami et al. [28], who applied their previously published [20] pipeline to map RNA-seq reads. Their dataset includes canonical A-to-I sites and non-canonical sites, validated or not, in repetitive and non-repetitive regions, resulting in about 1 million sites, most of them identified in the brain. The second and third [24] datasets were created by identifying RNA editing candidates using an analysis of transcriptome and genome sequencing data of a lymphoblastoid cell line from the same individual, revealing 147,029 and 446,670 sites, respectively. The fourth dataset was downloaded from DARNED [47]. All datasets were merged into single dataset containing $1,432,744$ unique editing sites.

The mouse RNA editing dataset was created by merging five datasets; the main dataset was created by Danecek et al., who screened for RNA-DNA differences, using whole brain RNA-seq analysis in 15 different mouse strains [4].

The second dataset published by Lagarrigue et al., investigated for RNA-DNA differences in liver and adipose tissues revealing 63 and 188 editing candidates, respectively [49]. The third dataset consisted of $176 \mathrm{~A}$ to I editing candidates, expressed in cerebral cortex [50]. The fourth dataset included 24 validated mouse A to I sites [48]. The fifth dataset was downloaded from DARNED [47].

\section{Zebrafish (ZF) editing dataset}

This dataset was created by analysis of RNA-seqs (SRA Accession numbers: SRR1028002, SRR1028003, SRR1028004). Fastq files were aligned to the ZF genome (Zv9/DanRer7) using tophat, command: tophat -r 530 index fastq1, fastq1_replication fastq2,fastq2_replication fastq3,fastq3_replication. We then realigned the fastq files to the zebrafish reference genome, and added the splice junction file, achieved from the first run, as input. 
Command: tophat -r 530 -j splice_junctions_file index fastq1,fastq1_replication fastq2,fastq2_replication fastq3, fastq3_replication.

Mpileup was then used to find RNA to reference-genome differences. Only sites with more than five edited reads and editing levels higher than 0.01 were taken into account.

\section{cDNA SNPs dataset}

Human cDNA SNPs were downloaded from dbSNP137 (All SNPs table), using the UCSC table browser [52]. We made a filter for SNPs that were annotated as 1 nucleotide length cDNA SNPs only, revealing 79,152 cDNA SNPs.

\section{Aligning editing sites flanking regions between species}

The sequences surrounding each editing site were downloaded using the UCSC table browser (versions: human-GRCh37/hg19, mouse-NCBI37/mm9). We used several sequence sizes and compared them by calculating signal-to-noise ratio, using SNP conservation as a control (Additional file 2: Figure S5). We chose to use a sequence of 40 nucleotides both upstream and downstream to the editing site, resulting in an 81 nucleotide sequence for each editing site. Nucleotide-nucleotide blast [51] (version: Nucleotide-Nucleotide BLAST 2.2.25+) was used to perform interspecies sequence alignment. Typical command: blastn -query organism1_dadaset.fa -strand 'both' -db organism2_blastdb -out out_file -task 'dc-megablast'.

We then defined a 70 identity threshold to ensure either high identity levels or long alignment length. Additionally, we restricted all hits to have an exact match on the edited nucleotide (the edited nucleotides from each species are matched and aligned).

Mouse editing and expression levels were obtained from a previously published study [4]. For each site we used the mean of its editing levels among all strains as published. Expression levels were calculated by reads count, using RNA-seq data from the strain with the highest reads depth (NOD_ShiLt)).

\section{Finding editing levels in human body map}

We aligned the Human-body-map dataset to the human genome (hg19), using Bowtie aligner [71] with liberal parameters that allow mismatch detection $(-n 3,-120$, -k 20, -e 140 -best). Following alignment, we collected all mismatches between the above reads to the reference genome. Mismatches in read positions with quality Phred score $<30$ were discarded. Editing levels was measured as \# of $G$ reads/\# of $A+G$ reads.

\section{Editing levels in rat, cow, opossum, and platypus}

Each RNA-seq was aligned to the matched reference genome (rat-rn4, cow-BosTau7, opossum-momDom5, and platypus-ornAna1) using STAR aligner with default parameters. Mismatches were screened using the same procedure as with human body map. Accession numbers for RNAseq: cow- SRR594491, rat- SRR594419, opossumSRR306744, SRR306746, platypus- SRR306727, SRR306729. Human body map- GSE7905.

Sequence logos were generated for $10 \mathrm{nt}$ upstream and downstream to the editing sites using WebLogo. [72].

RNA secondary structures were predicted using mfold [57].

Codon changes were calculates using ANNOVAR [73].

\section{Additional files}

\begin{abstract}
Additional file 1: Table S1. Additional information on the ESS, including human and mouse coordinates, year of publication, if they were found using next generation sequencing data analysis and molecular type of SNPs found in this position. Table S2. Additional sites that were found to be conserved by UCSC liftOver. Table S3. Editing profiles for the ESS in rat, cow, opossum, and platypus. For each organism we provide the matched nucleotide, number of $A+G$ reads, and editing levels. Table S4. Editing levels among 16 Human body map tissues. Table S5. Enriched GO terms in the ESS. Table S6. Human editing sites which harbor ' $G$ ' in mouse genome. Table S7. ESS sites that were found to be edited in zebrafish; coordinates are in Zv9/DanRer7 genome version. Table S8. Sequence conservation among mouse, dog, opossum, chicken, and zebrafish. Table S9. Editing levels among 16 Human body map tissues for human specific sites (deleted regions in mouse). Table S10. List of all non-conserved non-synonymous sites. Table S11. List of predicted complementary region for each site in the ESS.
\end{abstract}

Additional file 2: Figure S1. Incidence of editing sites per strain. The prevalence of editing sites was measured for the ESS (conserved sites) and all other sites ( $P$ value $=7.24 \times 10-10$, Student's t-test). Figure S2. Spatial proximity of conserved sites. The secondary structure shows spatial proximity of the conserved sites of (A) gria3, and (B) five intronic sites in the gria4 gene. Editing sites are depicted in orange and marked by an arrow. Figure S3. Conserved editing sites in microRNAs. Editing sites in pre-mir (A). The editing site is located in the seed region of mir376c. (B) Editing site within mir27b. Editing sites are highlighted in orange and marked by an arrow. Figure S4. Editing levels are conserved between human and mouse. RNA editing levels were measured in both human and mouse brains. We found positive correlation between editing levels in both species by calculating Pearson's correlation coefficient $(R=0.55)$. Figure $\mathbf{S 5}$. Signal-to-noise ratios. Signal-to-noise was measured by the ratio of editing hits to normalized SNPS hits. Both were calculated using the pipeline as described in the paper. We used $40 \mathrm{nt}, 80 \mathrm{nt}$, and $100 \mathrm{nt}$ blast alignment length and the UCSC liftover.

\section{Abbreviations}

ADAR: Adeonsine deaminase acting on RNA; BLAST: Basic local alignment tool; DARNED: Database of RNA editing; ECS: Exon Complementary Sequence; ESS: Evolutionary selected set; GLUR2: Glutamate receptor2; GO: Gene ontology; LINE: Long interspersed nuclear element; miRNA: microRNA; SNP: Single nucleotide polymorphism; UTR: Untranslated region.

\section{Competing interests}

The authors declare that they have no competing interests.

\section{Authors' contributions}

YP performed the research, analyzed the data, and wrote the manuscript. HYC participated in the design of the study and writing. EYL conceived the study, helped to analyze the data, and to write the manuscript. All authors read and approved the final version of the manuscript. 


\section{Acknowledgments}

We thank Jin Billy Li for comments on the manuscript, Yarden Opatowsky for helpful discussion, Lily Bazak and Miri Danan for help in data analysis, Orshay Gabay for graphical help and useful comments, Sol Efroni and Lior Appelbaum for data sharing and Shoshana Naiman for discussion. This work was supported by the European Research Council [311257] and by the Legacy Heritage Biomedical Science Partnership, Israel Science Foundation (grant numbers 1466/10), and by the I-CORE Program of the Planning and Budgeting Committee and the Israel Science Foundation (grant No 41/11).

Received: 18 September 2013 Accepted: 7 January 2014

Published: 7 January 2014

\section{References}

1. Bass BL: RNA editing by adenosine deaminases that act on RNA. Annu Rev Biochem 2002, 71:817-846.

2. Nishikura K: Functions and regulation of RNA editing by ADAR deaminases. Annu Rev Biochem 2010, 79:321-349.

3. Savva YA, Rieder LE, Reenan RA: The ADAR protein family. Genome Biol 2012, 13:252.

4. Danecek P, Nellaker C, McIntyre RE, Buendia-Buendia JE, Bumpstead S, Ponting CP, Flint J, Durbin R, Keane TM, Adams DJ: High levels of RNA-editing site conservation amongst 15 laboratory mouse strains. Genome Biol 2012, 13:26.

5. Rodriguez J, Menet JS, Rosbash M: Nascent-seq indicates widespread cotranscriptional RNA editing in Drosophila. Mol Cell 2012, 47:27-37.

6. Garrett S, Rosenthal JJ: RNA editing underlies temperature adaptation in $\mathrm{K}+$ channels from polar octopuses. Science 2012, 335:848-851.

7. Rosenthal JJ, Bezanilla F: Extensive editing of mRNAs for the squid delayed rectifier $\mathrm{K}+$ channel regulates subunit tetramerization. Neuron 2002, 34:743-757.

8. Polson AG, Bass BL, Casey JL: RNA editing of hepatitis delta virus antigenome by dsRNA-adenosine deaminase. Nature 1996, 380:454-456.

9. Morse DP, Bass BL: Long RNA hairpins that contain inosine are present in Caenorhabditis elegans poly(A) + RNA. Proc Natl Acad Sci USA 1999, 96:6048-6053.

10. Lev-Maor G, Sorek R, Levanon EY, Paz N, Eisenberg E, Ast G: RNA-editingmediated exon evolution. Genome Biol 2007, 8:R29.

11. Rueter SM, Dawson TR, Emeson RB: Regulation of alternative splicing by RNA editing. Nature 1999, 399:75-80.

12. Athanasiadis A, Rich A, Maas S: Widespread A-to-I RNA editing of Alu-containing mRNAs in the human transcriptome. PLoS Biol 2004, 2:e391

13. Scadden AD: The RISC subunit Tudor-SN binds to hyper-edited double-stranded RNA and promotes its cleavage. Nat Struct Mol Biol 2005, 12:489-496

14. Scadden AD, Smith CW: RNAi is antagonized by $A->$ I hyper-editing. EMBO Rep 2001, 2:1107-1111

15. Samuel CE: RNA editing minireview series. J Biol Chem 2003, 278:1389-1390.

16. Prasanth KV, Prasanth SG, Xuan Z, Hearn S, Freier SM, Bennett CF, Zhang $M Q$, Spector DL: Regulating gene expression through RNA nuclear retention. Cell 2005, 123:249-263.

17. Yang W, Chendrimada TP, Wang Q, Higuchi M, Seeburg PH, Shiekhattar R, Nishikura K: Modulation of microRNA processing and expression through RNA editing by ADAR deaminases. Nat Struct Mol Biol 2006, 13:13-21.

18. Kawahara $Y$, Zinshteyn $B$, Sethupathy $P$, lizasa $H$, Hatzigeorgiou AG, Nishikura K: Redirection of silencing targets by adenosine-to-inosine editing of miRNAs. Science 2007, 315:1137-1140

19. Godfried Sie C, Hesler S, Maas S, Kuchka M: IGFBP7's susceptibility to proteolysis is altered by A-to-I RNA editing of its transcript. FEBS Lett 2012, 586:2313-2317.

20. Ramaswami G, Lin W, Piskol R, Tan MH, Davis C, Li JB: Accurate identification of human Alu and non-Alu RNA editing sites. Nat Methods 2012, 9:579-581.

21. Li JB, Levanon EY, Yoon JK, Aach J, Xie B, Leproust E, Zhang K, Gao Y, Church GM: Genome-wide identification of human RNA editing sites by parallel DNA capturing and sequencing. Science 2009, 324:1210-1213.

22. Eisenberg E, Li JB, Levanon EY: Sequence based identification of RNA editing sites. RNA Biol 2010, 7:248-252.

23. Peng Z, Cheng $Y$, Tan BC, Kang L, Tian Z, Zhu Y, Zhang W, Liang Y, Hu X Tan X, Guo J, Dong Z, Liang Y, Bao L, Wang J: Comprehensive analysis of RNA-Seq data reveals extensive RNA editing in a human transcriptome. Nat Biotechnol 2012, 30:253-260.
24. Bahn JH, Lee JH, Li G, Greer C, Peng G, Xiao X: Accurate identification of A-to-I RNA editing in human by transcriptome sequencing. Genome Res 2012, 22:142-150.

25. Lin W, Piskol R, Tan MH, Li JB: Comment on "Widespread RNA and DNA sequence differences in the human transcriptome". Science 2012, 335:1302. author reply 1302.

26. Pickrell JK, Gilad Y, Pritchard JK: Comment on "Widespread RNA and DNA sequence differences in the human transcriptome". Science 2012, 335:1302. author reply 1302.

27. Kleinman CL, Majewski J: Comment on "Widespread RNA and DNA sequence differences in the human transcriptome". Science 2012, 335:1302. author reply 1302 .

28. Ramaswami G, Zhang R, Piskol R, Keegan LP, Deng P, O'Connell MA, Li JB: Identifying RNA editing sites using RNA sequencing data alone. Nat Methods 2013, 10:128-132.

29. Neeman $Y$, Levanon $E Y$, Jantsch MF, Eisenberg E: RNA editing level in the mouse is determined by the genomic repeat repertoire. RNA 2006, 12:1802-1809

30. Graveley BR, Brooks AN, Carlson JW, Duff MO, Landolin JM, Yang L, Artieri CG, van Baren MJ, Boley N, Booth BW, Brown JB, Cherbas L, Davis CA, Dobin A, Li R, Lin W, Malone JH, Mattiuzzo NR, Miller D, Sturgill D, Tuch BB, Zaleski C, Zhang D, Blanchette M, Dudoit S, Eads B, Green RE, Hammonds A, Jiang $L$, Kapranov $P$, et al: The developmental transcriptome of Drosophila melanogaster. Nature 2011, 471:473-479.

31. Levanon EY, Eisenberg E, Yelin R, Nemzer S, Hallegger M, Shemesh R, Fligelman ZY, Shoshan A, Pollock SR, Sztybel D, Olshansky M, Rechavi G, Jantsch MF: Systematic identification of abundant A-to-l editing sites in the human transcriptome. Nat Biotechnol 2004, 22:1001-1005.

32. Kim DD, Kim TT, Walsh T, Kobayashi Y, Matise TC, Buyske S, Gabriel A: Widespread RNA editing of embedded alu elements in the human transcriptome. Genome Res 2004, 14:1719-1725.

33. Blow M, Futreal PA, Wooster R, Stratton MR: A survey of RNA editing in human brain. Genome Res 2004, 14:2379-2387.

34. Wang Q, Miyakoda M, Yang W, Khillan J, Stachura DL, Weiss MJ, Nishikura K: Stress-induced apoptosis associated with null mutation of ADAR1 RNA editing deaminase gene. J Biol Chem 2004, 279:4952-4961.

35. Higuchi M, Maas S, Single FN, Hartner J, Rozov A, Burnashev N, Feldmeyer D, Sprengel R, Seeburg PH: Point mutation in an AMPA receptor gene rescues lethality in mice deficient in the RNA-editing enzyme ADAR2. Nature 2000, 406:78-81.

36. Galeano F, Tomaselli S, Locatelli F, Gallo A: A-to-I RNA editing: the "ADAR" side of human cancer. Semin Cell Dev Biol 2012, 23:244-250.

37. Horsch M, Seeburg PH, Adler T, Aguilar-Pimentel JA, Becker L, Calzada-Wack J, Garrett L, Gotz A, Hans W, Higuchi M, Holter SM, Naton B, Prehn C, Puk O, Racz I, Rathkolb B, Rozman J, Schrewe A, Adamski J, Busch DH, Esposito I, Graw J, Ivandic B, Klingenspor M, Klopstock T, Mempel M, Ollert M, Schulz $H$, Wolf $E$, Wurst W, et al: Requirement of the RNA-editing enzyme ADAR2 for normal physiology in mice. J Biol Chem 2011, 286:18614-18622.

38. Feldmeyer D, Kask K, Brusa R, Kornau HC, Kolhekar R, Rozov A, Burnashev N, Jensen V, Hvalby O, Sprengel R, Seeburgh RH: Neurological dysfunctions in mice expressing different levels of the Q/R site-unedited AMPAR subunit GluR-B. Nat Neurosci 1999, 2:57-64.

39. Gurevich I, Tamir H, Arango V, Dwork AJ, Mann JJ, Schmauss C: Altered editing of serotonin $2 \mathrm{C}$ receptor pre-mRNA in the prefrontal cortex of depressed suicide victims. Neuron 2002, 34:349-356.

40. Sommer B, Kohler M, Sprengel R, Seeburg PH: RNA editing in brain controls a determinant of ion flow in glutamate-gated channels. Cell 1991, 67:11-19.

41. Niswender CM, Copeland SC, Herrick-Davis K, Emeson RB, Sanders-Bush E: RNA editing of the human serotonin 5-hydroxytryptamine $2 \mathrm{C}$ receptor silences constitutive activity. J Biol Chem 1999, 274:9472-9478.

42. Iwamoto K, Kato T: RNA editing of serotonin $2 \mathrm{C}$ receptor in human postmortem brains of major mental disorders. Neurosci Lett 2003, 346:169-172.

43. Schellekens H, Clarke G, Jeffery IB, Dinan TG, Cryan JF: Dynamic 5-HT2C receptor editing in a mouse model of obesity. PLoS One 2012, 7:e32266.

44. Bhalla T, Rosenthal JJ, Holmgren M, Reenan R: Control of human potassium channel inactivation by editing of a small mRNA hairpin. Nat Struct Mol Biol 2004, 11:950-956.

45. Hoopengardner B, Bhalla T, Staber C, Reenan R: Nervous system targets of RNA editing identified by comparative genomics. Science 2003, 301:832-836. 
46. Reenan RA: Molecular determinants and guided evolution of species-specific RNA editing. Nature 2005, 434:409-413.

47. Kiran AM, O'Mahony JJ, Sanjeev K, Baranov PV: Darned in 2013: inclusion of model organisms and linking with Wikipedia. Nucleic Acids Res 2013, 41:D258-D261.

48. Maas S, Godfried Sie CP, Stoev I, Dupuis DE, Latona J, Porman AM, Evans B, Rekawek P, Kluempers V, Mutter M, Gommans WM, Lopresti D: Genome-wide evaluation and discovery of vertebrate A-to-I RNA editing sites. Biochem Biophys Res Commun 2011, 412:407-412.

49. Lagarrigue $S$, Hormozdiari F, Martin $\amalg$, Lecerf F, Hasin Y, Rau C, Hagopian R, Xiao Y, Yan J, Drake TA, Ghazalpour A, Eskin E, Lusis AJ: Limited RNA editing in exons of mouse liver and adipose. Genetics 2013, 193:1107-1115.

50. Dillman AA, Hauser DN, Gibbs JR, Nalls MA, McCoy MK, Rudenko IN, Galter $D$, Cookson MR: mRNA expression, splicing and editing in the embryonic and adult mouse cerebral cortex. Nat Neurosci 2013, 16:499-506.

51. Altschul SF, Gish W, Miller W, Myers EW, Lipman DJ: Basic local alignment search tool. J Mol Biol 1990, 215:403-410.

52. Hinrichs AS, Karolchik D, Baertsch R, Barber GP, Bejerano G, Clawson H, Diekhans M, Furey TS, Harte RA, Hsu F, Hillman-Jackson J, Kuhn RM, Pedersen JS, Pohl A, Raney BJ, Rosenbloom KR, Siepel KE, Sugnet CW, Sultan-Qurraie A, Thomas DJ, Trumbower H, Weber RJ, Weirauch M, Zweig AS, Haussler D, Kent WJ: The UCSC Genome Browser Database: update 2006. Nucleic Acids Res 2006, 34:D590-D598.

53. Zhu H, Urban DJ, Blashka J, McPheeters MT, Kroeze WK, Mieczkowski $P$ Overholser JC, Jurjus GJ, Dieter L, Mahajan GJ, Rajkowska G, Wang Z, Sullivan PF, Stockmeier CA, Roth BL: Quantitative analysis of focused a-to-I RNA editing sites by ultra-high-throughput sequencing in psychiatric disorders. PLoS One 2012, 7:e43227.

54. Merkin J, Russell C, Chen P, Burge CB: Evolutionary dynamics of gene and isoform regulation in Mammalian tissues. Science 2012, 338:1593-1599.

55. Barbosa-Morais NL, Irimia M, Pan Q, Xiong HY, Gueroussov S, Lee LJ, Slobodeniuc V, Kutter C, Watt S, Colak R, Kim T, Misquitta-Ali CM, Wilson MD, Kim PM, Odom DT, Frey BJ, Blencowe BJ: The evolutionary landscape of alternative splicing in vertebrate species. Science 2012, 338:1587-1593.

56. Aruscavage PJ, Bass BL: A phylogenetic analysis reveals an unusual sequence conservation within introns involved in RNA editing. RNA 2000, 6:257-269.

57. Zuker M: Mfold web server for nucleic acid folding and hybridization prediction. Nucleic Acids Res 2003, 31:3406-3415.

58. Higuchi M, Single FN, Kohler M, Sommer B, Sprengel R, Seeburg PH: RNA editing of AMPA receptor subunit GluR-B: a base-paired intron-exon structure determines position and efficiency. Cell 1993, 75:1361-1370.

59. Herb A, Higuchi M, Sprengel R, Seeburg PH: Q/R site editing in kainate receptor GluR5 and GluR6 pre-mRNAs requires distant intronic sequences. Proc Natl Acad Sci USA 1996, 93:1875-1880.

60. Kuehbacher A, Urbich C, Zeiher AM, Dimmeler S: Role of Dicer and Drosha for endothelial microRNA expression and angiogenesis. Circ Res 2007, 101:59-68.

61. Chen WJ, Yin K, Zhao GJ, Fu YC, Tang CK: The magic and mystery of microRNA-27 in atherosclerosis. Atherosclerosis 2012, 222:314-323.

62. Eisenberg E, Adamsky K, Cohen L, Amariglio N, Hirshberg A, Rechavi G, Levanon EY: Identification of RNA editing sites in the SNP database. Nucleic Acids Res 2005, 33:4612-4617.

63. Gommans WM, Tatalias NE, Sie CP, Dupuis D, Vendetti N, Smith L, Kaushal R, Maas S: Screening of human SNP database identifies recoding sites of A-to-I RNA editing. RNA 2008, 14:2074-2085.

64. Eggington JM, Greene T, Bass BL: Predicting sites of ADAR editing in double-stranded RNA. Nat Commun 2011, 2:319.

65. Lehmann KA, Bass BL: Double-stranded RNA adenosine deaminases ADAR1 and ADAR2 have overlapping specificities. Biochemistry 2000 39:12875-12884

66. Greenberger S, Levanon EY, Paz-Yaacov N, Barzilai A, Safran M, Osenberg S, Amariglio N, Rechavi G, Eisenberg E: Consistent levels of A-to-I RNA editing across individuals in coding sequences and non-conserved Alu repeats. BMC Genomics 2010, 11:608.

67. Rosenthal JJ, Seeburg PH: A-to-I RNA editing: effects on proteins key to neural excitability. Neuron 2012, 74:432-439.

68. Schmitz J, Brosius J: Exonization of transposed elements: a challenge and opportunity for evolution. Biochimie 2011, 93:1928-1934.
69. Garbern JY, Neumann M, Trojanowski JQ, Lee VM, Feldman G, Norris JW, Friez MJ, Schwartz CE, Stevenson R, Sima AA: A mutation affecting the sodium/proton exchanger, SLC9A6, causes mental retardation with tau deposition. Brain 2010, 133:1391-1402.

70. Gommans WM, Mullen SP, Maas S: RNA editing: a driving force for adaptive evolution? Bioessays 2009, 31:1137-1145.

71. Langmead B, Trapnell C, Pop M, Salzberg SL: Ultrafast and memory-efficient alignment of short DNA sequences to the human genome. Genome Biol 2009, 10:R25.

72. Crooks GE, Hon G, Chandonia JM, Brenner SE: WebLogo: a sequence logo generator. Genome Res 2004, 14:1188-1190.

73. Wang K, Li M, Hakonarson H: ANNOVAR: functional annotation of genetic variants from high-throughput sequencing data. Nucleic Acids Res 2010, 38:e164.

doi:10.1186/gb-2014-15-1-r5

Cite this article as: Pinto et al:: Mammalian conserved ADAR targets comprise only a small fragment of the human editosome. Genome Biology 2014 15:R5.

\section{Submit your next manuscript to BioMed Central and take full advantage of:}

- Convenient online submission

- Thorough peer review

- No space constraints or color figure charges

- Immediate publication on acceptance

- Inclusion in PubMed, CAS, Scopus and Google Scholar

- Research which is freely available for redistribution

Submit your manuscript at www.biomedcentral.com/submit
C BioMed Central 